\title{
Rule Chaining in MARVEL: Dynamic Binding of Parameters
}

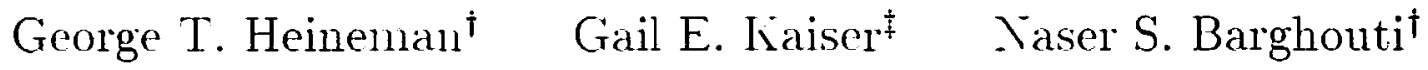 \\ Israel Z. Ben-Shaul ${ }^{\S}$
}

\author{
CUCS-(022-91 \\ May 6, 1991
}

\begin{abstract}
MARVEL is a rule-based dovelopment environmen (RBDE) that assists in the development of software projects. MARVL encapsulates each software development activity in a rule that specifies the condition for invohing the aclivity and its effects on the components of the project under development. These components are abstracted as objects and stored in a persistent object database. Wach rule applies to a specific class of objects. which is specified as the parameter of the rule. Firing a rule entails binding its formal parameter to a specific object. If the rule clinges the object in such a way that the conditions of other rules become satisfied, these other rules are nutomatically fired. A problem arises in this forward chaining model when the classes of the objects manipulated by the rules are different. MARVEL has to determine which object to bind to the parameter of each rule in the chain. based on the object manipulated by the original rule that initiated the chain. We describe a heuristic approach for solving this problem in the current MARVEL implementation and introduce an algorithmic approach that does better.
\end{abstract}

Keywords: Programming-in-1, he-large. reasoning techniques, lifecycle support, experience report.

(c) 1991, Heineman. Kaiser, Barghouti, Ben-Shaul

\footnotetext{
${ }^{\dagger}$ Barghouti and Heineman are supported in part by the Center for 'lelecommunications Research.

thaiser is supported by National Science Foundation granls CCR-9000930, CDA-8920080 and CCR-8858029, by grants from ATIT. BAR. DEC.IBM. SIRA and Xerox, by the New York State Center for Advanced Technology on Computer and Information Systems and b: the NSF Engineering Research Center for Telecommunications Research.

3en-Shaul is supported in part by the Center for Advanced Technology.
} 


\section{Introduction and Motivation}

Soltware development environments (SDEs) assist developers of software projects in organizing the project 's data and carrying out the development. process. Every software project assumes a specific organization for its components, and a specific derelopment process that might be different from other projects. Thus. it is not appropriate to build a single software development environment, with a hardwired assistance model. Instead. the assistance should be knowledgebased. In order to provide intelligent isssistance, an SIDE needs specifications of two things: the project's data model and the project's development process.

MARVEL is an instance of a particular class of SDEs, called rule-based development environments (RBDEs), which use a rule-based model of the development process. A project administrator writes a specification of the project's process model and loads it into Marvel. Marvel then tailors its functionality accordingly and presents the user with a choice of commands that correspond to the loaded rules. M.ABVil, provides automated assistance by applying forward and backward chaining anong the rules in order to automatically invoke activities that are parts of the development process.

One distinguishing feature of MARTLL is its integration of object-oriented data modeling and rule-based process modeling [2]. Fach development activity on the components of the project under development is modeled as a rule that specifies the condition for invoking the activity and the effect of the ardivity on the components. The project's components are modeled as objects and stored in a persistent object clablyase. The attributes of each object and relationships to other objects are defined by its class. The rules are treated as methods of these classes; in particular. each rule has a formal parameter whose type is a specific object class ${ }^{1}$.

When a user requests a command. the corresponding rule is invoked. The formal parameter of the rule is bound to the object selected by the user. For example, the user might wish to edit

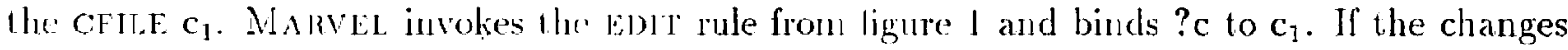
that the rule introduces to this object cause the condition of other rules to become satisfied, MARVEL automatically fires each of these rules and attempts to bind their formal parameters to actual objects. MARVEL must infer which objects to bind to the formal parameters of the rules in the chain. The selcetion of these objects is a difficult problem when a rule that is a method of one class chains to a rule that is a melhorl of another class, and thus a different object must be bound to the formal parameter of the second rule. In addition, recursive data definitions cause chaining between rules that act on different objects within the same class. We call the problem of binding parameters of rules in a rule chain the chaining problem.

In this paper. we describe the chaining problem and explain how it is solved in the current implementation of MARVEL. We describe MARVEL, detailing only those aspects necessary to understand the chaining problem and un solutions; for a more complete description of MARVEL, sec $[12.13,11]$. We next explain the $\cdot$ laining problem in detail with a motivating example. We describe a heuristic approach to solving this problem and discuss its implementation in the current MARVEL system. We then extend the example to show the limitations of this approach,

\footnotetext{
${ }^{1}$ For this paper. we restrict rules to hav only one formal parameter. We are investigating chaining between multi-method rules.
} 


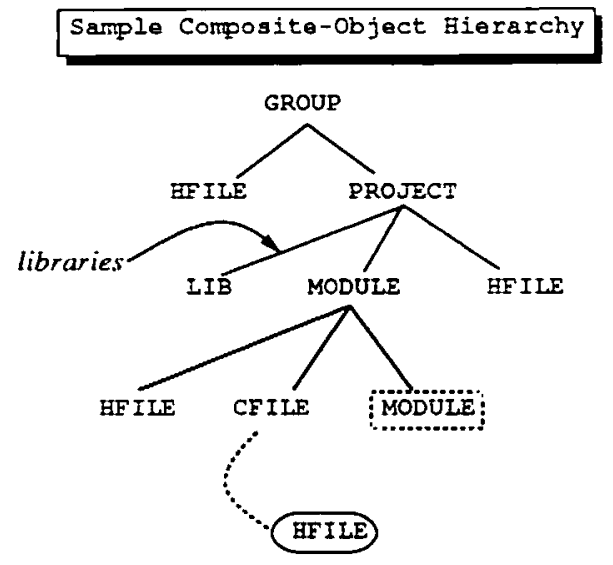

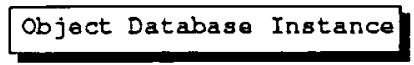

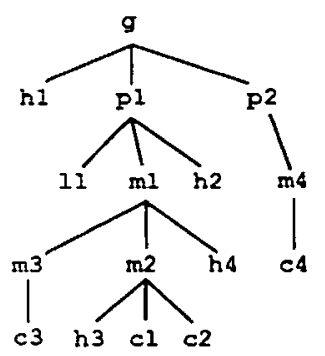

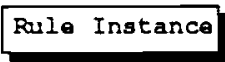

Edit [?c:CEILE] :

(?c.rcs = Reserved)

$\{$ edit ?c \}

(?c.status $=$ Modified)

Figure 1: MARVEL data and process model

and present a betier algorithmir solution. We conchurle with related work on RBDEs and other kinds of rule-based systems. and summarize our contributions.

\section{Marvel Overview}

MARVEL is an RIBDE which allows a project administrator to create and tailor an environment by defining a data model and a precess model in the MSL specification language. Software artifacts (i.e. code. documentation) are abstracted as instances of classes. which are defined in the data model. and stored in an objet database. The attributes of each object are defined by its class: for example. a PRO.JECT object has a set attribute libraries which contains objects from the LIB class. For clarily, the names of wher attributes are not shown in figure 1 . The set attributes create a compositr-object hicrarchy. ligure 1 depicts in sample composite-object hierarchy and an object database instance for this hicrarcliy. Eacls object in this example belongs to the class with the same first letter. Note how the object $\mathrm{g}$ is composed of an instance of class HFILE $\left(\mathrm{h}_{1}\right)$ and two instances of class PROIECT $\left(\mathrm{p}_{1}\right.$ and $\left.\mathrm{p}_{2}\right)$. Essentially, the composite-object hierarchy is an "IS-PART" relationship. MARVEL also provides for links from one object to another. For example, in figure 1 , the class CFIL has a link attribute, includes (dashed line), to an HFILE. This link specilies that an instance of the CFILE is associated with a particular HFILE. Links and composite-objects enhance the power of the Mst rule language by allowing queries in the rules to traverse the database structure: we term sucl queries as navigational.

The process model is defined by rules that specify a condition that must be satisfied for the rule to fire, and offects that assert changes on the object database. The collection of all the rules for a certain project is termed the rulebase of that project. Since rules in MaRvEL are methods on objects, they have a formal parameter with which the rule is invoked. Rules also have derived parameters which are the result of queries made by the rule on the object database. The query language consists of boolenn combinations of three navigational primitives, member, linkto and ancrstor and standard relational operators (i.e. $\leq .=$ ) which allow the query to navigate through the object database. Member finds the parent/child of an object, ancestor 
finds ancestors/descendents (in the composite-object hierarchy) for a given object, and linkto finds objects that are linked to a given object.

For example, the TOUCH [?M:MODUL, rule from figure 2 requires an object from the MODULE class as the formal parameter, and makes a query on the object database to determine two derived parameters. ? $\mathrm{r}$ is defined as "a MODULE that is a member of the modules set attribute of ? $\mathrm{m}$ and whose stutus attibute is Modified" and "a CFILE that is a member of the cfiles attribute of ?m and whose statu: attribute is Modified." When quantified with forall $(\forall)$, the derived parameter is bound to all the objects returned by the query, while exists $(\exists)$ binds the parameter to the first such object found.

The condition of a MANVEL rule determines the logical state of the object database necessary for a given invocation to fire. For exampla. in figure 1 the intended CFILE object must be reserved (rcs attribute set to Reserved) befor coliting. The conditions are constructed from predicates based on the rule's parameters (formal and derived). which must be satisfied for the rule to fire. If a predicate in the condition is false, it is said to be unsatisfied. and backward chaining could be automatically intiated on this failed predicate in an at tempt to satisfy it; this is similar to attempting to achieve a subgoal.

Each MaRVEL rulc has a set of (possibly) multiple. mut wally exclusive effects that are asserted on the object database upon completion of the rule. For example. the EDIT rule in figure 1 asserts that the CFILE object has been modified (stalus attribute is set to Modified). Once an effect is asserted on the object datillatse, forward chaining could be initiated to automatically fire other rules. A forward chain from one rule to another is initiated if the effect of the first rule makes an assertion which satislies the condition of the second. We now present the two basic forms of chaining. backuard and foruard. as they operate in MARVEL.

\subsection{Backward Chaining}

In MARVEL. when the user invokes a rule $r$ whose condition is not satisfied, backward chaining is initiated on a failed predicate $p$ in the condition to attempt to satisfy it. The system collects together into a set $S_{b}$ those rules in the rulebase that have an effect which satisfies $p$. The system then repeatedly remores anul invokes $r_{i}$ from this set until either $p$ is satisfied, or $S_{b}$ is empty: Since backward chaining is a recursive process, the system might initiate a backward chain to satisfy $r_{i}$. Becauso the condition is a combination of predicates. satisfying $p$ might still not satisfy the entire condition for $r$. so this backward chaining process is repeated until the condition is satisfied, or all known possibilities are cxhausted.

Note that the chaining problem appears when invoking $r$ since it has a formal parameter that the system must automatically determine. In the current MARVEL implementation, however, we bypass this problem since we restrict effects to make assertions only on the formal parameter, not derived ones. 


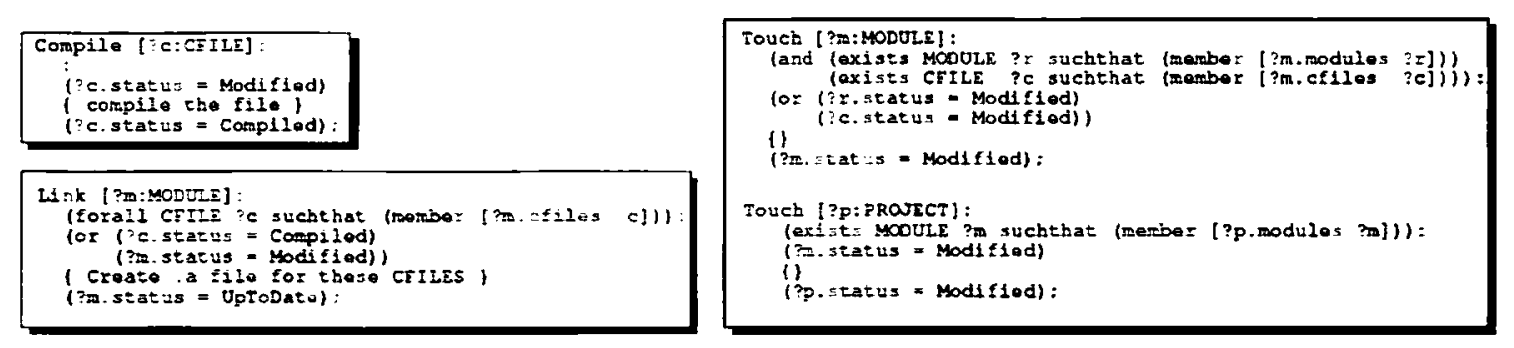

Figure 2: Motivating Fxample

\subsection{Forward Chaining}

Forward chaining is initiated when a predicate $p$ in the effect of a rule is asserted on the object database. MARVLI, determines those rules $r_{;} \in S_{j}$ in the system whose condition might become satisfied because of this assertion. This is a tenuous connection since the condition of rule $r_{i}$ might be composed of many predicales. and the system has chosen only one of these as a reason to forward chain: however. this provirles . MARVEL with an automatic way to logically determine the flow of control in the system.

Since the condition of a rule can involve derjed parameters, the chaining problem appears and cannot be bypassed. Specifically, in rule $r_{i}$. the predicate $p$ might be based on a derived parameter, and hile system must dedcrmine the formal parameter with which to invoke $r_{i}$. In this paper we are only concened with forward chaining, since in our current model, backward chaining exhibits no problems. The actual details of the problems arising during forward chaining will be elaborated on in the next scotion.

\section{Chaining Problem}

In a rule-based system. when the system determines that it must chain to rule $r_{i}$, it must determine which object to bind to $r_{i}$ s formal parancter; this is the essence of the chaining problem. The reader should observe that if there were no derived parameters, this problem would not exist, for the conditions and effects of a rule would be based solely on the formal parameter.

MARVEL, like AP5 [4] and other systems, allows for rules to have derived parameters by using existential and universal operators to bind quantified parameters to certain objects based on arbitrary logical expressions. Figure I shows some rules in the MsL language. In this example. if the TOLCH[?M:MODLLE] me were invoked on object $m_{2}$ from figure 1 , MARVEL would bind ?c to either $c_{1}$ or $c_{2}$ (whicherer has a stalus attribute value of Modified), and ?r would be cmpty since there is no child of m.2 of he MODELE class.

The ability to make querjes gives the rules more power (since their activities are not limited to formal parameters). but it complicates the chaining mechanism. Consider a forward chain to $r_{i}$ based on a predicate with a derived parameter. In order for the system to fire rule $r_{i}$, it must determine the object to bind to the formal parameter. For example. if the EDIT rule from 
figure 1 is involed on $c_{1}$. then a forward chain is initialed to TOUCH[?M:MODULE], but it is not readily apparent 1, what object ?n should be bound as there exists four module objects. In the next section. we explore several ways to solve the chaining problem, and describe the current MARVEL implementation.

\section{Heuristic Approach}

There are several possible ways to retermine the objects to bind to the formal parameter of a forward-chained rule.

- Manual: The systen could ask the user for the specific object to invoke the rule with. The main drawback to this policy is that the usor should not need to know the details of rules and chaining. In addition. the user might respond incorrectly, causing the system to perform unnecessary activities and fail to enact the proper activities.

- Logical: Since the derived parameters are logically determined, it might be possible to invert this logic to determine the proper object to use. We discuss this possibility in more detail in the algorithmic approach section.

- Heuristics: l'he systen can use heuristics to search for the proper object. If the system assumes that rule chaining orcurs in a localized area in the object database, then the search space can be kept small. Is an alternative to searching: the system could also use a set of axions (as put forth in cirAPPlE [10]) to determine legal choices for the parameter.

In MARVEL we have implemented a set of heuristics to search "near" an object to determine the proper objects to use during chaining. In doing so. MARVEL makes use of the composite-object hierarchy, since it assumes that objects near each ot her in the hierarchy are semantically related. The search path also include links (screction 2) since they also define semantic associations between objects. During a forwarl chain to rule $r_{i}$ from a rule invoked with object $O$, we search for the object to bind to $r_{i}$ s formal parameter in the following order:

1. The object $O$ itself.

2. O's parent object.

3. $O^{\circ}$ s immediate children.

4. The objects associated (throngh links) with $O$.

j. $\mathrm{O}^{\circ}$ s proper ancestors.

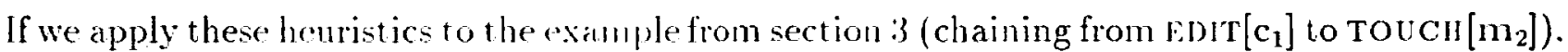
we see that step 2 dedermines the cormel object. To give a more involved example. if TOUCH[ $\left.\mathrm{m}_{2}\right]$

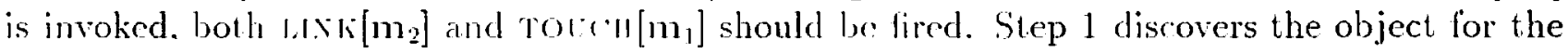
LINK rule. while step 2 tales care of roucil. The heuristic, obriously, cannot stop at the first candidate found, but must collect all candiclates together since it is possible to have multiple instantiations of the same rule with different objects as well as different rules with same or different objects. 


\section{Implementation}

This scction briefly describes the static and dynamic support currently implemented in MARVEL to provide lor chaining. Since the data and process models are tailorable. MARvel reads in their specification, as written in the Msı. language by the administrator of the environment. The system compiles a ruk netuork, an efficient data structure which maintains the chaining possibilitics (both backward and forward) for each predicate in each rule. This rule network is stored on disk upon completion for later invocations of VARVEL. Because this is a static process, it is executed only when either model changes.

The runtime support is provided by the Opportumist. which is MARVEL's chaining engine. When the user issues a command. the Opport unist matches this to a rule $r$ in the rulebase. The user selects the argument from the object database and the system invokes $r$. First. MARVEL generates the derived parameters by sequentially eraluating each of the quantified expressions in $r^{\circ}$ s condition. Next. MARYEI. craluates the predicates that must be true for $r$ to fire. If there is a predicate $p$ that is not satisfied. then MARVEL collects from the rule network those rules $r_{i}$ that have an effect which satisfy this predicate. MARVEL invokes these rules until the set is exhausted or the predicate is satisfied. MARVEL binds the failed object to $r_{i}$ s formal parameter since effects are restricted to be based on the formal parameter.

Once r's condition is satisfied. MARVEL executes $r$ 's activity with the necessary information (see [i] for a full description of this process) and selects the proper effect to assert as determined by the result of 1.his execution. For every predicate $p$ in this effect, MARvel asserts it on the object database and collects those rules from the rule network that may now be satisfied. MARVEL enters the forward chaining cycle. and automatically invokes each of these rules $r_{i}$ in turn, potentially generating more rules to invoke in a recursive manner.

To invoke these pules. MarVF. must determine the object to bind to $r_{i}$ 's formal parameter from the predicate $p$. If the object $o_{i}$ in $p$ is of the proper class, then MARVEL binds the formal parameter to $o_{i}$, otherwise MARIEL performs a search from the object bound to $r$ 's formal parameter based upon the heuristics described in section 4. If MARVEL is unable to find one, then it does not invoke the rule.

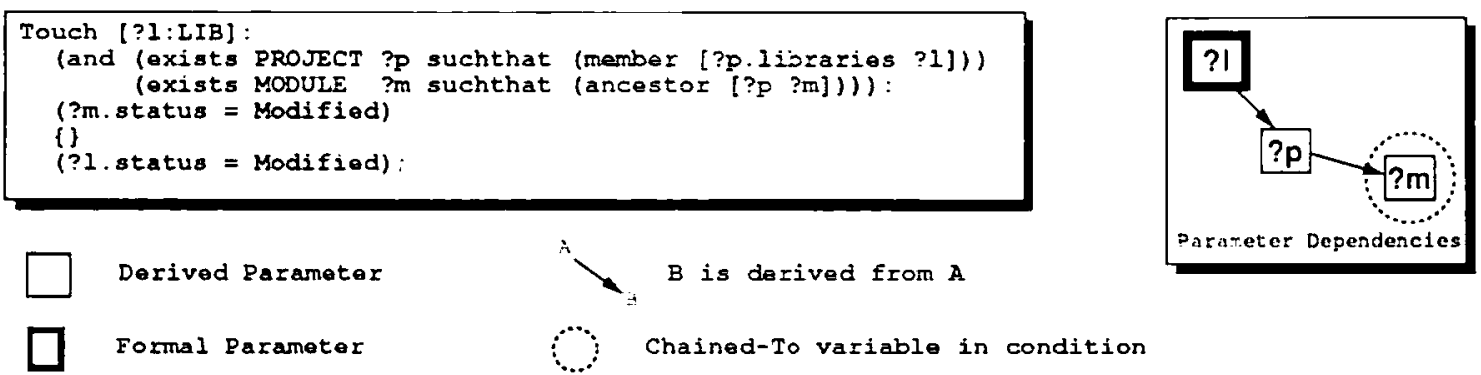

Figno 3: Extending the example from figure 2 


\section{Algorithmic Approach}

If we extend the example from figure 2. our heuristic approach fails to generate the proper formal parameter to use for chaining; we add the TotCn[? I.LIB] rule shown in figure 3 . This rule queries the object database for the PROJECT object ?p which contains this LIB ?I and then finds a MODULE object ? $m$ which is a descendent of ?p that is Modified. If ? $\mathrm{m}$ is found, then ?l is marked as Modified. If roc $\mathrm{CH}\left[\mathrm{m}_{1}\right]$ is invoked and succeeds. $\left(\mathrm{m}_{1}=\right.$ Modified) is asserted and MARVFI. initiates a forward chain to TOUCH[?L:LIB]. The heuristic approach outlined in section 4 will fail to detcrmine the formal parameter since $l_{1}$ is a sibling of $m_{1}$ (see figure 1 ), and falls outside the search space: for any fixed searching heuristic, there will always be cases which fail. The algorithmic approach, however, will be able to correctly invert the derived parameters to gencrate the possibilities for chaining.

The intuition behind the algorithmic approach is that each navigational query (from section 2) is itself invertible. For example. in figure 3. (exists PROJECT ?p suchthat (member

[?p.libraries ?1])) returus the parent(s) of the object(s) bound to ?l. This can be inverted to bind to ? the children objects. in the libraries attribute, of the object(s) bound to ?p.

We outline our algorithm in figure 4. We assume that each rule has a dependency graph which determines the dependencies betweol parameters in a given rule. In figure 3 , for example, ? $m$ is dependent upon ?p which is rependent upon ?l. This information is statically determined from the derived parameters. Issume hat MARVEL initialces a forward chain from rule $r$ to rule $r_{i}$ because a predicate $p$ in $r$ saltisfies a predicate $q$ in $r_{i}$ s condition. The object $O$ changed by $p$ is subsequently bound to the parameter. ?V. that q operates on. If ?V is the formal parameter of $r_{i}$, then the system invokes $r_{i}$ on $O$. Otherwise. ?V is a clerived parameter.

The algorithm inverts each parameter in the dependency graph, starting from ?V, until the formal parameter is determined. The Augment procedure in figure 4 reverse-evaluates the expression of a quantified parameter to determine objects to which other parameters can be bound. For example, line $\bar{i}$ in Augment covers the example on member mentioned earlier this section. If the formal parameter remains undetermined. then the system cannot chain to $r_{i}$; in contrast. failure to find an object to bind to the formal parameter using the heuristic approach, might only mean that the hemristic is too weak.

In figure 5 we see the results of the forward chain after MARVEL has involed TO: $\mathrm{CH}\left[\mathrm{m}_{1}\right]$ from figure 2. When the predicate $(? \mathrm{~m}$. status $=$ Modified) is asserted on the object database, the system forward chains to the TOlCH[?L:LIB] rule from figure 3 . To determine the object to bind to the formal parameter. the system inverts the expression which defines the parameter ? $\mathrm{m}$. Initially, $\mathrm{m}$ is bound to $\mathrm{m}_{1}$. Figure 5 outlines the progress of the algorithm. giving key values at the specified lines. Note how the algorithm inverts the expressions for ? $m$ and $? p$ in order. as it proceeds to determine ?l. After the first call to Augment), $\mathrm{p}_{1}$ is added to the list of objects bound to ?p (in line 8 ). The second iteration inverts the expression for ?p and binds ?1 to $l_{1}$. This retermines the formal paranetcr. and Touch is instantiated for $l_{1}$.

It might not be clear why his logical inversion process is preferred to searching the object database. Since the expression for a parameter can be arbitrarily complicated. any heuristic, aside from searching the entire object database, will potentially fail to find certain objects 
?Par.objects is the set of objects that are bound to ?Par.

?T.value is the set of parameter augmentations generated by the AUgment procedure for a given expression.

?F is the formal parameter of the chained-to rule.

$S$ is a stack of parameters to be inverted.

ALGORITHM Invert[?V]

1. IF (?V is the formal parameter ?F) THEN

2. Add [?F.objects, 0]

ELSE

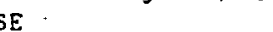

3. $S=\{? V\} ;$ ? .objects $=\{0\}$;

4. WHILE $S$ not empty DO

5. $\quad$. $\quad$ V $=$ POP $(S)$;

6. IF (?V = ?F) THEII CONTINUE; \{Skip if this is the formal parameter\}

\{ The expression for the derived parameter ?V is a quantification of an arbitrary expression $T$. \}

7. Augment [T, ?V] :

8. Evaluate[T.value];

$\{$ For every (derived) parameter that has had their set of possible objects augmented by the \}

$\{$ previous function, push onto the stack $S$.

9. FOR every parameter ?P in $T$.value DO

10. PUSH(?P):

END:

11. Instantiate the rule $R$ for each object from the ?F.object list.

END; f of ALGORITHM Invert[?V] \}

Wi is an arbitrary expression representad by an AlD/OR tree.

?V is the parameter being quantified in this expression

?Par is the "other" parameter in the navigational primitive expression (primitives are binary)

PROCEDURE Augment [N, ?V]

1. FOR all children $C$ of $\|$ DO

2. Augment $[\mathrm{C}, ? \mathrm{~V}]$ :

3. IF ( $I$ is an internal node) THE

CASE H OF

4. AND: I.value = Intersection of C.value FORALL children $\mathbf{C}$ of $\mathbb{W}$.

5. OR: M.value = Union of C.value FORALL children $C$ of $B$.

EILD:

ELSE

\{ Hote that $H$ is a navigational primitive. ?V is the (known) parameter in this primitive \}

( which is being quantified. ?Par is tha other (unknown) parameter in the primitivo \}

CASE $\triangle$ OF

Member [?V.attr?Par]: $\quad\{$ ? is a parent of ?Par $\}$

6. N.value = Add[?Par.objects,objects in (?V.objects).attr];

Nember [?Parattr ?V]: $\quad$ [?V is a child of ?Par $\}$

7. H.value = Add[?Par.objects,parent object of (?V.objects)]:

Linkto [?V.attr ?Par]: \{?V links to ?Par \}

8. N.value = Add[?Par,objects,objects from (?V.objects),attr that linkto an object of the ?Par class]

Linkto [?Par.attr ?V]: \{?V is linked from ?Par \}

9. H.value = Add[?Par.objects,objects from the ?Par class that linkto (?V.objects) through the "attr" attribute]

Ancestor [?V ?Par]: $\quad\{$ ? is an ancestor of ?Par $\}$

10. I. value = Add[?Par.objects, objects from the ?Par class that are a descendant from (?V.objects)]

Ancestor [?Par ?V] : $\quad\{? V$ is a descendant of ?Par $\}$

11. H.value = Add[?Par.objects, objects from the ?Par class that are an ancestor of (?V.objects)]

EHD;

END; \{ of PROCEDURE Augment [u,?V]\}

Figure 4: Inwerting Derived Parameters Algorithm 

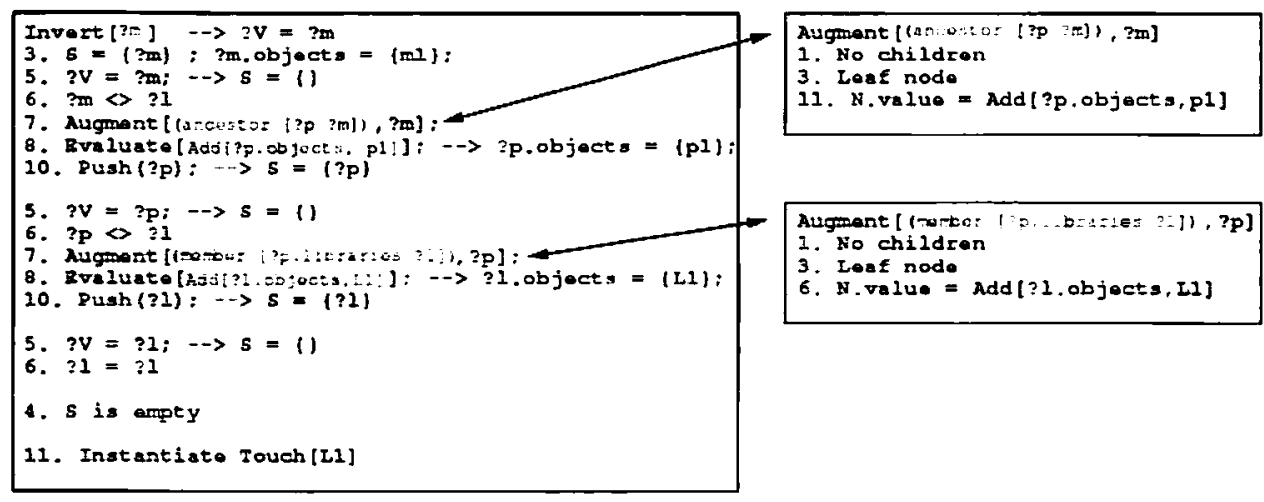

Figure 5: Solving the chain for figure 3

which could be bound to the formal parameter. In MARVEL. and RBDFs in general, the object database might contiain several thousand. and possibly more objects. It would be extremely inefficient to perform a search on the cntire object database. We are currently investigating the implementation of the logic-based inversion method.

\section{Related Work}

There are many other systems that support intelligenl assistance: we focus on those systems that execute some form of chaining 10 perform this assistance.

EPOS [14] and TPLAX [6] attemp to achieve subgoals (backward chaining) when the condition for an involied rule is not satisfied. Darwin [15] carries out inferencing to determine whether or not an activity is allowed. Theso kind of systems perlorm strictly backward chaining, and are usually Prolog-based. The unification scheme in Prolog successfully detcrmines parameters to match because the body of Prolog clauses have no quanlified expressions like those in MsL. If MARVEL allowed effects to be asserterl on derived parameters. then backward chaining would also be affected by the chaining problem. As it stands. however. MARVEL's backward chaining cycle, like Prolog unification. is straight forward. Oikos [1] extends a Darwin-like system to more complicated control structures among rules, bu1 uses a blackboard scheme that passes all necessary information from one hackboard to another dicectly thus bypassing the chaining problem.

The MERLIN [9] system has both backward and forward chaining capabilities, but it avoids the chaining problem: backward chaining is resolved through Prolog-style unification, while forward chaining explicitly lists the paraneters (much like a blackboard scheme) that are passed from one rule to the nex1. ALF [S] has forward chaining capabilities, but the paper does not state how it determines paraneters. GRAlPl, [10] is exceptional, in that it explicitly addresses the parameter issue that arises in chainiug between rules.

Odin [3], AP5 [-1] and OPS5 sistem have rules that are triggers activated when the state of the object database changes. The chaining problem does not appear under this data-driven 
approach. since there is no specificd llow of control. Rule-based database systems like POSTGRlis [16] work in a similar fashion.

The composite-object hierarchy allows MARVEL to make efficient navigational queries to the object database: in addition. the algorithmic approach outlined in section 6 is dependent on this structure. since this makes an efficient inversion process. The PROBE DBMS [5] provides for queries on object databases. and outlines some methods for optimization. If a system has relations, instead of structure. that determine the interconnections between the object cntities (like in EPOS) then these navigational queries can also be implemented, but will only be as officient as the system's query optimizer.

\section{Contributions}

In the current implementation of MARVLL we address the chaining problem by using a fixed set of heuristics to search lor the proper parameter. Our heuristics secmed satisfactory for many cases. However. as we enhanced the XISL rule language and began experimenting with more intricate rules, chaining cases arose that escaped our heuristics. One possibility would be to increase the breadth of the searching heuristics, these cases can be more efficiently solved by logically inverting the derived parameters. We have given an algorithm that performs this inversion process for an arbitrarily complex MSL rule.

\section{References}

[1] V. Ambriola, I'. Ciancarini. and Montanegro ( $\therefore$ Sofiware Process Enactment in Oikos. In Fourth AC:M SICSOFT Symprosimm on Soffuar Development Environments. pages 183192, Irvine CA. December 19\%1.

[2] Naser S. Barghouti and Gail F. Kaiser. Modeling (oncurrency in Rule-Based Development Fnvironments. IEEE Expe\% j(6):15-2i. December 1990.

[3] G. M. Clemm. The Workshop System - A Practical Knowledge-Based Software Enviromment. In ACM SICSOFT/SICPLAN Softwar Engineering Symposium on Practical Sofluar Devtopment Enciromments, pages 55-64, Boston. November 1988. ACM Press. Special issue of SICPLAN Sotires, 2.4(2). February 1989.

[4] D. Cohen. Compiling complex database transition triggers. In 1989 ACM SIGMOD International Confrence on the Management of Data, pages 225-234, Portland OR, June 1989. Special issue of $S / G M O D$ Rtrord. $18(2)$. June 1989.

[5] Umeshwar Jayal t al. Simplifying complex objects: The ProBE approach to modeling and querying them. In Stanley B. Zdonili and David Maier, editors, Readings in Object-Oriented Database Systems. pages 390-399. Morgan Kaufmann, Inc., 1990. 
[6] Deboral Frincke. Myla Archer. and Kärl Levitt. A Planning System for the Intelligent Testing of Secure Softrare. In hinowledge-Based Softwar Assistant KBSA-5, pages 346360 , Liverpool NY. September 1990.

[1] Mark (iisi and Gail I.. Kaiser. Extending A Tool Integration Language (Experience Report). Technical Report (UiCS-014-91. Columbia University Department of Computer Science. April 1991. Submitted for publication.

[8] C. Godart, F. Charoy, and .J.C. Demiame. Computer Assisted Software Engineering: Characterisation and Modeling. IC' $/ 89$ - Toronto. 1989.

[9] H. Hünnekens and C. Junkermann and B. Peuschel and IV. Schäfer, J. Vagts. A Step Towards Knowledgo-beased Software Process Modeling. In 1st Conference on System Development Enviromments and Factorics (SDEQF 1). Pitman Publishing, London, 1990.

[10] K. E. Huff and V.R. I.esser. A Plan-based Intelligent Assistant that Supports the Software Development Process. In ACI/S/CiSOFT/SICPLAN Softure Engineering Symposium on Practicul Softure 1) velopment limvivoninents, pages 9i-106. Boston. MA, November 1988. Special issue of SICPLAN Votirts. 24(2). February 1989.

[11] Gail E. Kaiser. Naser S. Barghouti. Peter H. Feiler, and Robert W. Schwanke. Database Support for Knowledge-Based Lingincering Environments. IEEE Expert. 3(2):18-32, Summer 1988.

[12] Gail E. Kaiser. Naser S. Barghouti, and Michael H. Sokolsky. Experience with Process Modeling in the Marvel Software Development Environment Kernel. In Bruce Shriver, editor. 23rd Annual Hawaii Intermational Conference on System Sciences, volume II, pages 131-140. Kona. HI. January logu.

[13] Gail E. Kaiser. Peter II. Feiler. and Steven S. Popovich. Intelligent Assistance for Software Development and Maintenance. HEEE Softure. 5(3):40-49, May 1988.

[14] Chunnan Liu. Software Process Panning and Fxecution: Coupling vs. Integration. Technical report, Norwegian Institule of Technology (NTH), November 1990.

[15] N. H. Minsky and D. Rozenshtcin. A Software Development Environment for Law-Governed Systems. In ACM SICSOFT/SICPLAI Softuare Engineering Symposium on Practical Softuare Development Rnvironments. pages 6.5-75. Boston MA. November 1988.

[16] Wichael Stonebraker and lawrence A. Rowe. The Design of POSTGRFs. In Carlo Zaniolo, editor. 1989 ACH STCHMOD Intermational Conference on the Management of Data, pages 340-35.5. Washington DC, May 1986. Special issue of SIGMOD Record, 18(2), June 1989. 